\title{
Leishmaniasis among neighbouring endemic countries in the Eastern Mediterranean, African and European regions
}

Citation: Leishmaniasis among neighbouring endemic countries in the Eastern Mediterranean, African and European regions. East Mediterr Health J. 2019;25(1):66-68 https://doi.org/10.26719/2019.25.1.66

Copyright $@$ C World Health Organization (WHO) 2019. Some rights reserved. This work is available under the CC BY-NC-SA 3.o IGO license (https:// creativecommons.org/licenses/by-nc-sa/3.o/igo).

\section{Introduction}

The World Health Organization (WHO) Eastern Mediterranean Region reported $69.6 \%$ of the total number of cutaneous leishmaniasis cases detected worldwide in 2016 (1). Over 90\% of reported cases in the Region were from three countries only: Afghanistan, Pakistan and Syrian Arab Republic. Moreover, the number of reported cases in Syrian Arab Republic and the neighbouring countries of Iraq, Jordan and Lebanon, indicated a remarkable increase between 2010 and 2016 (1). For visceral leishmaniasis, the Region carries about $19 \%$ of the global burden, with the highest number of cases reported from Somalia and Sudan (1).

The Organization has strengthened its efforts in leishmaniasis prevention and control in the Region in recent years, producing a framework for action on cutaneous leishmaniasis in the Eastern Mediterranean Region 2014-2018, and a regional manual for case management of cutaneous leishmaniasis (2).

However, the Region faces many challenges in implementing the control measures for leishmaniasis, including uncontrolled urbanization, limited funding, weak surveillance, and emergencies and crisis situations that have led to the deterioration of sanitation and water supply, collapse and destruction of health system infrastructure, and population displacement. Furthermore, endemic zones for cutaneous and visceral leishmaniasis traverse country borders and WHO regions, underlining the need for cross-border collaboration between neighbouring countries and WHO regions.

Given this background, WHO held an interregional meeting on leishmaniasis among neighbouring endemic countries in the WHO Eastern Mediterranean, African and European regions, in Amman, Jordan, from 23 to 25 September 2018.

The objectives of the meeting were to:

- review epidemiology and control of leishmaniasis in countries of the Eastern Mediterranean Region, as well as neighbouring countries in the African and European Regions;

- discuss the strategic elements and operational action required to enhance early diagnosis and treatment, including surveillance and data management/report- ing, access to medicines and consumables, control of vectors and reservoir hosts, and capacities of health staff;

- share experiences on surveillance of leishmaniasis with emphasis on the District Health Information Software (DHIS2) online tool;

- identify ways to address and overcome the challenges faced by countries in controlling the disease, notably in complex operational environments;

- identify cross-border issues and areas of collaboration to improve disease control; and

- agree on priority research topics for leishmaniasis within the universal health coverage agenda.

The meeting was attended by representatives from the ministries of health of Albania, Georgia, Greece, Islamic Republic of Iran, Iraq, Jordan, Lebanon, Morocco, Pakistan, Saudi Arabia, Sudan, Syrian Arab Republic, and Tunisia. The WHO Secretariat included staff from headquarters, regional and country levels.

Dr Hoda Atta, Coordinator HIV, TB, Malaria and Tropical Diseases, WHO Regional Office for the Eastern Mediterranean, Cairo, Egypt, inaugurated the meeting by welcoming the participants and acknowledging its importance, given the current socio-political context and related challenges for neglected tropical diseases in the three regions.

\section{Summary of discussions}

Weak surveillance and underreporting are key challenges. High-burden countries need to ensure that they enter data onto the WHO District Health Information System 2 (DHIS2) platform. It is important to establish unified case definitions when reporting and to differentiate between autochthonous and imported cases in order to identify the most probable sources of infection.

Global, regional and country level experience in responding to outbreaks of leishmaniasis was presented. WHO is currently supporting countries endemic for visceral leishmaniasis with donations of medicines and/ or funds for control programmes through agreements and donations from Gilead Sciences (GILEAD) and the United Kingdom's Department for International Development (DfID). Extensions of these agreements are

\footnotetext{
This report is extracted from the Summary report on the Interregional meeting on leishmaniasis among neighbouring endemic countries in the Eastern Mediterranean, African and European regions, Amman, Jordan, 23-25 September 2018 (http://applications.emro.who.int/docs/IC_Meet Rep_2019_EN_22325.pdf?ua=1).
} 
dependent on the effective use and reporting of activities by control programmes. For cutaneous leishmaniasis, there are currently no medicine donations and only limited funding from WHO.

The global leishmaniasis programme at WHO headquarters has an emergency warehouse stocked with medicines and diagnostics that has very limited capacity. Priority is given to visceral leishmaniasis over cutaneous leishmaniasis due to the fatal nature of the disease if left untreated. This limited capacity greatly hinders the capability of the warehouse to respond to emergency requests from countries, especially in cases of cutaneous leishmaniasis. There is a need for high level advocacy in order to ensure access to treatment for all those affected. Countries should consider procurement through national resources or partner support.

Challenges for vector control include inadequate infrastructure for vector surveillance and control, lack of standardized vector and reservoir control measures, lack of monitoring and evaluation of WHO-interventions, insufficient human capacity and capability, limited contribution of research to the development of vector control policies and strategies, and poor community engagementand healtheducation messaging. Hence, there is a dire need to strengthen the capacity of health staff in skin neglected tropical diseases, including leishmaniasis, and address poor compliance with recommended diagnostic methods for cutaneous leishmaniasis. This includes capacity-building in diagnosis and treatment (for health professionals), surveillance to align with WHO global reporting requirements (for programme officers/data managers), vector and reservoir control (for entomologists and mammologists), and reinforcing programme management (for managers of control programmes). WHO Collaborating Centres have a key role to play in providing expert technical support, guidance and information exchange.

Moreover, countries face many external challenges that hinder the effective implementation of interventions, including ongoing insecurity in some areas, population movement, inadequate financial resources, high trained staff turnover, and weak intersectoral collaboration. The diversity of the disease and associated vectors and animal reservoir hosts, coupled with these external challenges, underpins the need for standardization and clarity of guidance.

In addition, to enhance cross-regional collaboration it is important to have uniform reporting mechanisms for disease surveillance, sharing relevant research findings, and using standardized case management protocols. There is also a need for mapping at subnational levels, especially in bordering areas, in order to inform both national and local coordination.

\section{Recommendations}

1. Implementing resolution WHA60.13 on Control of leishmaniasis (3) to ensure that:

- domestic funds are allocated for the procurement of first-line treatment for both cutaneous and visceral leishmaniasis; and

- human leishmaniasis is included in the national surveillance system so that countries can collect and analyse routine data.

2. Building/sustaining political commitment to ensure uninterrupted implementation of activities for prevention and control of leishmaniasis.

3. Developing/updating national programmes and strategies on prevention and control of leishmaniasis, using a multisectoral approach and community engagement, and protocols for case management of leishmaniasis, to ensure compliance with WHO recommendations.

4. Promoting cross-border collaboration and cooperation on leishmaniasis prevention and control among neighbouring countries of the WHO Eastern Mediterranean, African and European regions.

5. Identifying, in collaboration with WHO, research priorities for prevention and control of leishmaniasis, and support the relevant research activities.

6. Providing technical support to countries to implement web-based surveillance of leishmaniasis within the national health information system.

7. Creating and funding a central procurement mechanism to promptly ship medical supplies (medicines and rapid diagnostic tests) on emergency or routine basis to selected countries where WHO has temporarily taken over this function on behalf of the health ministry.

8. Continuing to support capacity-building for the prevention, control and surveillance of leishmaniasis and to publish self-learning training packages, online and offline, on visceral leishmaniasis (Leishmania infantum) and cutaneous leishmaniasis (African and Asian forms).

9. Continuing to facilitate intercountry and interregional coordination on prevention and control of leishmaniasis.

10. Developing guidance outlining core interventions for emergency and complex control situations. 


\section{References}

1. World Health Organization. Leishmaniasis: key facts. Geneva: World Health Organization; 2018 (https://www.who.int/newsroom/fact-sheets/detail/leishmaniasis).

2. World Health Organization Regional Office for the Eastern Mediterranean. Framework for action on cutaneous leishmaniasis in the Eastern Mediterranean Region 2014-2018. Cairo: World Health Organization Regional Office for the Eastern Mediterranean; 2014 (http://applications.emro.who.int/dsaf/EMROPUB_2013_EN_1591.pdf?ua=1).

3. Sixtieth World Health Assembly. WHA60.13 control of leishmaniasis. Geneva: World Health Organization; 2007 (https://www. who.int/neglected_diseases/mediacentre/WHA_60.13_Eng.pdf). 